\title{
Continuous functions between sets with operations
}

\author{
Fumie NAKaOKa ANd Nobuyuki Oda
}

\begin{abstract}
A set with an operation is a generalization of a topological space. Two types of continuous functions are defined between sets with operations. They are characterized making use of two types of closures and interiors. Homeomorphisms between sets with operations are also characterized. Variants of subspaces, connected spaces and compact spaces are introduced in a set with an operation and some fundamental properties of them are proved.
\end{abstract}

\section{Introduction}

In a previous paper [6] we introduced $a$ set with an operation as a generalization of a topological space (see Section 2); let $\mathcal{P}(X)$ be the power set of a set $X$ and $\mathcal{F} \subset \mathcal{P}(X)$ such that $\mathcal{F}$ contains at least one non-empty set. An operation $\kappa$ on $\mathcal{F}$ is a function

$$
\kappa: \mathcal{F} \rightarrow \mathcal{P}(X)
$$

such that $U \subset U^{\kappa}$ for each $U \in \mathcal{F}$, where $U^{\kappa}=\kappa(U)$. We call the triple $(X, \mathcal{F}, \kappa)$ a space. A topological space $(X, \tau)$ with a family $\tau$ of open sets is regarded as a space $(X, \tau$, id), where id: $\tau \rightarrow \mathcal{P}(X)$ is defined by $\operatorname{id}(U)=U$ for any $U \in \tau$ (see Example 2.2). The $\kappa$-operation is a generalization of the operations for topological spaces (called $\alpha$ - or $\gamma$-operations) studied by Kasahara [4], Janković [3] and Ogata [7]. However, the $\kappa$-operation and the space $(X, \mathcal{F}, \kappa)$ defined above are used to generalize the concept of "topological space" itself, that is, for a space $(X, \mathcal{F}, \kappa)$, variants of open set, neighborhood, interior, closure etc. can be defined without assuming the existence of topological spaces [6]. For example, for a space $(X, \mathcal{F}, \kappa)$, the $\kappa$-open set is defined and the family of all $\kappa$-open sets is denoted by $\mathcal{F}_{\kappa}$ (see Definition 2.1). The purpose of the present paper is to define two kinds

Received September 2, 2019

2010 Mathematics Subject Classification. 54A05.

Key words and phrases. Continuous, operation.

https://doi.org/10.12697/ACUTM.2020.24.15 
of continuous functions $f:(X, \mathcal{F}, \kappa) \rightarrow(Y, \mathcal{G}, \psi)$ between sets with operation $(X, \mathcal{F}, \kappa)$ and $(Y, \mathcal{G}, \psi)$, that is, $(\kappa, \psi)$-continuous (Definition 3.1) and $\left(\mathcal{F}_{\kappa}, \mathcal{G}_{\psi}\right)$-continuous (Definition 3.2) functions and study their properties. The functions $f:(X, \tau) \rightarrow\left(Y, \tau^{\prime}\right)$ between topological spaces with operation were studied by Kasahara [4], Janković [3], Ogata [7], Rosas and Vielma [8] and Basu et al. [1], for example.

We defined two types of closures $\left(\mathrm{Cl}_{\kappa}, \mathcal{F}_{\kappa}-\mathrm{Cl}\right)$ and two types of interiors $\left(\operatorname{Int}_{\kappa}, \mathcal{F}_{\kappa}\right.$-Int) for a space $(X, \mathcal{F}, \kappa)$ in [6] (see Section 2). The $(\kappa, \psi)$ continuous functions $f:(X, \mathcal{F}, \kappa) \rightarrow(Y, \mathcal{G}, \psi)$ are characterized making use of $\mathrm{Cl}_{\kappa}, \mathrm{Cl}_{\psi}, \mathrm{Int}_{\kappa}$, Int $\psi$ by Theorem 3.6; the $\left(\mathcal{F}_{\kappa}, \mathcal{G}_{\psi}\right)$-continuous functions are characterized making use of $\mathcal{F}_{\kappa^{-}} \mathrm{Cl}, \mathcal{G}_{\psi^{-}} \mathrm{Cl}, \mathcal{F}_{\kappa^{-}}$-Int, $\mathcal{G}_{\psi^{-}}$-Int by Theorem 3.9. Therefore, Theorem 3.7 shows that if $f:(X, \mathcal{F}, \kappa) \rightarrow(Y, \mathcal{G}, \psi)$ is $(\kappa, \psi)$ continuous, then $f$ is $\left(\mathcal{F}_{\kappa}, \mathcal{G}_{\psi}\right)$-continuous. In Section 3.1 we study homeomorphisms between sets with operation.

In Section 4 we consider subspaces. Let $(X, \mathcal{F}, \kappa)$ be a space and $S$ a subset of $X$. Let $\mathcal{F}_{\kappa} \mid S=\left\{U \cap S \mid U \in \mathcal{F}_{\kappa}\right\}$. Since the relation $U \cap S=V \cap S$ does not always imply $\kappa(U) \cap S=\kappa(V) \cap S$ (see Example 4.1), the function $\kappa_{S}: \mathcal{F} \mid S \rightarrow \mathcal{P}(S)$ is not well defined by the formula $\kappa_{S}(U \cap S)=\kappa(U) \cap S$ for any $U \cap S \in \mathcal{F} \mid S$. Therefore, we define a subspace of a space $(X, \mathcal{F}, \kappa)$ by the triple $\left(S, \mathcal{F}_{\kappa} \mid S\right.$, id) (see Definition 4.3$)$ and prove standard results. In the case of a topological space $(X, \tau)$, Rosas and Vielma [8] noticed that an operation on a subspace $\alpha_{S}: \tau_{S} \rightarrow \mathcal{P}(S)$ satisfying $\alpha_{S}(G \cap S)=\alpha(G) \cap S$ with $G \in \tau$ is not necessarily well defined in some cases and gave an example (see Remark 4.2).

We define the $\mathcal{F}_{\kappa^{-}}$connected space (Definition 5.1) and $\mathcal{F}_{\kappa^{-}}$-compact space (Definition 6.1), and study the relations with $\left(\mathcal{F}_{\kappa}, \mathcal{G}_{\psi}\right)$-continuous function $f:(X, \mathcal{F}, \kappa) \rightarrow(Y, \mathcal{G}, \psi)$ in Sections 5 and 6 , respectively.

\section{Sets with operations}

In this section we recall some results from [6] which are necessary for the subsequent discussions. Let $\mathcal{P}(X)$ be the power set of a set $X$ and $\mathcal{F} \subset \mathcal{P}(X)$ such that $\mathcal{F}$ contains at least one non-empty set. An operation $\kappa$ on $\mathcal{F}$ is a function

$$
\kappa: \mathcal{F} \rightarrow \mathcal{P}(X)
$$

such that $U \subset U^{\kappa}$ for each $U \in \mathcal{F}$, where $U^{\kappa}=\kappa(U)$. We call the triple $(X, \mathcal{F}, \kappa)$ a space. If $(X, \mathcal{F}, \kappa)$ is a space, then we call $X$ a $(\mathcal{F}, \kappa)$-space, or simply, a $\kappa$-space.

Definition 2.1 (see [6], Definition 2.2). Let $(X, \mathcal{F}, \kappa)$ be a space. A subset $A$ of $X$ is called a $\kappa$-open set of $X$ if for each $x \in A$ there exists a set $U \in \mathcal{F}$ such that $x \in U \subset U^{\kappa} \subset A$. The family of all $\kappa$-open sets is denoted by $\mathcal{F}_{\kappa}$. A subset $F$ of $X$ is called a $\kappa$-closed set of $X$ if its complement $X-F$ is a $\kappa$-open set in $X$. 
By [6, Proposition 2.4], a subset $A$ of $(X, \mathcal{F}, \kappa)$ is $\kappa$-open if and only if there exists an index set $\Lambda$ and $U_{\lambda} \in \mathcal{F}$ for each $\lambda \in \Lambda$ such that $A=$ $\cup_{\lambda \in \Lambda} U_{\lambda}=\cup_{\lambda \in \Lambda} U_{\lambda}^{\kappa}$.

Let $x$ be any point of $X$. A set $U^{\kappa}$ such that $x \in U \in \mathcal{F}$ is called a $\kappa$-neighborhood of $x$. A $\kappa$-open set $W$ such that $x \in W$ is called a $\kappa$-open neighborhood of $x$.

Example 2.2. Let $(X, \tau)$ be a topological space with the family $\tau$ of open sets of $X$. Let $\mathcal{F}=\tau$ and $\kappa: \mathcal{F}=\tau \rightarrow \mathcal{P}(X)$ be defined by the function $\kappa(U)=U$ (the same set) for any $U \in \tau$. Then $\mathcal{F}_{\kappa}=\tau$ and a $\kappa$-open set is an open set in $\tau$. Moreover, $\kappa$-neighborhoods and $\kappa$-open neighborhoods of a point $x$ are usual open neighborhoods of $x$ in the topological space $(X, \tau)$. Therefore, we regard a topological space $(X, \tau)$ as the set $X$ with $\mathcal{F}=\tau$ and the operation $\kappa(U)=U$ so that $\mathcal{F}_{\kappa}=\tau$ (if $\kappa$ is not defined otherwise), that is, a topological space $(X, \tau)$ is a space $(X, \tau$, id $)$, where id $: \tau \rightarrow \mathcal{P}(X)$ is defined by $\operatorname{id}(U)=U$ for any $U \in \tau$.

Definition 2.3 (see [6], Definition 3.2). Let $(X, \mathcal{F}, \kappa)$ be a space and let $A$ be a subset of $X$.

A point $x$ of $X$ is called a $\kappa$-interior point of $A$ if there exists $U \in \mathcal{F}$ such that $x \in U \subset U^{\kappa} \subset A$. The $\kappa$-interior of $A$ is defined by

$$
\operatorname{Int}_{\kappa}(A)=\{x \mid x \text { is a } \kappa \text {-interior point of } A\} .
$$

A point $x$ of $X$ is called a $\mathcal{F}_{\kappa}$-interior point of $A$ if there exists $V \in \mathcal{F}_{\kappa}$ such that $x \in V \subset A$. The $\mathcal{F}_{\kappa}$-interior of $A$ is defined by

$$
\mathcal{F}_{\kappa}-\operatorname{Int}(A)=\left\{x \mid x \text { is a } \mathcal{F}_{\kappa} \text {-interior point of } A\right\} \text {. }
$$

The following hold for any subset $A$ of $(X, \mathcal{F}, \kappa)$ by [6, Proposition 3.5].

(1) $A \supset \operatorname{Int}_{\kappa}(A) \supset \mathcal{F}_{\kappa}-\operatorname{Int}(A)$.

(2) If $A$ is a $\kappa$-open set, then $A=\operatorname{Int}_{\kappa}(A)=\mathcal{F}_{\kappa}-\operatorname{Int}(A)$.

Definition 2.4 (see [6], Definition 3.7). Let $A$ be a subset of $(X, \mathcal{F}, \kappa)$.

A point $x$ of $X$ is called a $\kappa$-adherent point of $A$ if $U^{\kappa} \cap A \neq \emptyset$ for any $U \in \mathcal{F}$ with $x \in U$, or there exists no $U \in \mathcal{F}$ with $x \in U$. The $\kappa$-closure of $A$ is defined by

$$
\mathrm{Cl}_{\kappa}(A)=\{x \mid x \text { is a } \kappa \text {-adherent point of } A\} .
$$

A point $x$ of $X$ is called a $\mathcal{F}_{\kappa}$-adherent point of $A$ if $V \cap A \neq \emptyset$ for any $V \in \mathcal{F}_{\kappa}$ with $x \in V$, or there exists no $V \in \mathcal{F}_{\kappa}$ with $x \in V$. The $\mathcal{F}_{\kappa}$-closure of $A$ is defined by

$$
\mathcal{F}_{\kappa^{-}} \mathrm{Cl}(A)=\left\{x \mid x \text { is a } \mathcal{F}_{\kappa^{-}} \text {-adherent point of } A\right\} .
$$

Although some points of $(X, \mathcal{F}, \kappa)$ may have no $\kappa$-neighborhoods or $\kappa$ open neighborhoods, the inclusions $A \subset \mathrm{Cl}_{\kappa}(A)$ and $A \subset \mathcal{F}_{\kappa}-\mathrm{Cl}(A)$ hold for any $A \subset X$ by Definition 2.4. 
We note that the following relations hold for any subset $A$ of $(X, \mathcal{F}, \kappa)$ :

$$
\begin{gathered}
\operatorname{Int}_{\kappa}(A) \subset \cup \mathcal{F} \text { and } \mathcal{F}_{\kappa}-\operatorname{Int}(A) \subset \cup \mathcal{F}_{\kappa} ; \\
\mathrm{Cl}_{\kappa}(A) \supset X-\cup \mathcal{F} \text { and } \mathcal{F}_{\kappa}-\operatorname{Cl}(A) \supset X-\cup \mathcal{F}_{\kappa} .
\end{gathered}
$$

The following hold for any subset $A$ of $(X, \mathcal{F}, \kappa)$ by [6, Proposition 3.11]:

(1) $A \subset \mathrm{Cl}_{\kappa}(A) \subset \mathcal{F}_{\kappa}-\mathrm{Cl}(A)$;

(2) if $A$ is a $\kappa$-closed set, then $A=\mathrm{Cl}_{\kappa}(A)=\mathcal{F}_{\kappa}-\mathrm{Cl}(A)$.

If $A$ is a subset of $(X, \mathcal{F}, \kappa)$, then $\mathcal{F}_{\kappa}$ - $\operatorname{Int}(A)$ is a $\kappa$-open set and $\mathcal{F}_{\kappa}$ - $\mathrm{Cl}(A)$ is a $\kappa$-closed set by [6, Proposition 3.14]. Moreover, we have the following results for subsets $A$ and $B$ of $(X, \mathcal{F}, \kappa)$ by [6, Propositions 3.16 and 3.18].

(1) $\mathcal{F}_{\kappa}-\operatorname{Int}\left(\mathcal{F}_{\kappa}-\operatorname{Int}(A)\right)=\mathcal{F}_{\kappa}$-Int $(A)$. If $A \subset B$, then $\mathcal{F}_{\kappa}-\operatorname{Int}(A) \subset \mathcal{F}_{\kappa}-\operatorname{Int}(B)$.

(2) $\mathcal{F}_{\kappa}-\mathrm{Cl}\left(\mathcal{F}_{\kappa^{-}} \mathrm{Cl}(A)\right)=\mathcal{F}_{\kappa}-\mathrm{Cl}(A)$. If $A \subset B$, then $\mathcal{F}_{\kappa}-\mathrm{Cl}(A) \subset \mathcal{F}_{\kappa}-\mathrm{Cl}(B)$.

(3) If $A \subset B$, then $\operatorname{Int}_{\kappa}(A) \subset \operatorname{Int}_{\kappa}(B)$ and $\mathrm{Cl}_{\kappa}(A) \subset \mathrm{Cl}_{\kappa}(B)$.

The following statements are equivalent for any subset $A$ of $(X, \mathcal{F}, \kappa)$ by [6, Proposition 3.22].

(1) $A$ is $\kappa$-open.

(2) $A=\operatorname{Int}_{\kappa}(A)$.

(3) $A=\mathcal{F}_{\kappa}-\operatorname{Int}(A)$.

(4) $\mathrm{Cl}_{\kappa}(X-A)=X-A$.

(5) $\mathcal{F}_{\kappa}-\mathrm{Cl}(X-A)=X-A$.

(6) $X-A$ is $\kappa$-closed.

Remark 2.5. Let $(X, \mathcal{F}, \kappa)$ be a space. Let $U_{1}, U_{2}, V_{1}, V_{2} \in \mathcal{F}$. We note that

(1) an equality $U_{1} \cup U_{2}=V_{1} \cup V_{2}$ does not always imply $U_{1}^{\kappa} \cup U_{2}^{\kappa}=$ $V_{1}^{\kappa} \cup V_{2}^{\kappa}$

(2) an equality $U_{1} \cap U_{2}=V_{1} \cap V_{2}$ does not always imply $U_{1}^{\kappa} \cap U_{2}^{\kappa}=$ $V_{1}^{\kappa} \cap V_{2}^{\kappa}$

(3) the equality $\left(U_{1} \cup U_{2}\right)^{\kappa}=U_{1}^{\kappa} \cup U_{2}^{\kappa}$ does not always hold;

(4) the equality $\left(U_{1} \cap U_{2}\right)^{\kappa}=U_{1}^{\kappa} \cap U_{2}^{\kappa}$ does not always hold.

For example, let $X=\{a, b, c, d\}$ and

$$
\mathcal{F}=\{\emptyset,\{a\},\{b\},\{c\},\{a, b\},\{b, c\},\{a, c\},\{a, b, c\}, X\} .
$$

Let $\kappa: \mathcal{F} \rightarrow \mathcal{P}(X)$ be an operation defined by

$$
\begin{gathered}
\emptyset^{\kappa}=\emptyset,\{a\}^{\kappa}=\{a\},\{b\}^{\kappa}=\{c\}^{\kappa}=\{b, c\}^{\kappa}=\{b, c\}, \\
\{a, b\}^{\kappa}=\{a, b, d\},\{a, c\}^{\kappa}=\{a, c, d\},\{a, b, c\}^{\kappa}=X^{\kappa}=X .
\end{gathered}
$$

(1) Let $U_{1}=\{a\}, U_{2}=\{b, c\}, V_{1}=\{a, b\}, V_{2}=\{c\} \in \mathcal{F}$. Then $U_{1} \cup U_{2}=$ $\{a\} \cup\{b, c\}=\{a, b\} \cup\{c\}=V_{1} \cup V_{2}$ and

$$
U_{1}^{\kappa} \cup U_{2}^{\kappa}=\{a\}^{\kappa} \cup\{b, c\}^{\kappa}=\{a\} \cup\{b, c\}=\{a, b, c\} ;
$$

$V_{1}^{\kappa} \cup V_{2}^{\kappa}=\{a, b\}^{\kappa} \cup\{c\}^{\kappa}=\{a, b, d\} \cup\{b, c\}=\{a, b, c, d\}=X$.

Therefore, we have $U_{1}^{\kappa} \cup U_{2}^{\kappa} \neq V_{1}^{\kappa} \cup V_{2}^{\kappa}$. 
(2) Let $U_{1}=\{a\}, U_{2}=\{a, b\}, V_{1}=\{a, b\}, V_{2}=\{a, c\} \in \mathcal{F}$. Then $U_{1} \cap U_{2}=\{a\} \cap\{a, b\}=\{a, b\} \cap\{a, c\}=V_{1} \cap V_{2}$ and

$$
\begin{gathered}
U_{1}^{\kappa} \cap U_{2}^{\kappa}=\{a\}^{\kappa} \cap\{a, b\}^{\kappa}=\{a\} \cap\{a, b, d\}=\{a\} ; \\
V_{1}^{\kappa} \cap V_{2}^{\kappa}=\{a, b\}^{\kappa} \cap\{a, c\}^{\kappa}=\{a, b, d\} \cap\{a, c, d\}=\{a, d\} .
\end{gathered}
$$

Therefore, we have $U_{1}^{\kappa} \cap U_{2}^{\kappa} \neq V_{1}^{\kappa} \cap V_{2}^{\kappa}$.

(3) Let $U_{1}=\{a\}, U_{2}=\{b, c\} \in \mathcal{F}$ as in (1). Then $\left(U_{1} \cup U_{2}\right)^{\kappa}=$ $\{a, b, c\}^{\kappa}=X$ and $U_{1}^{\kappa} \cup U_{2}^{\kappa}=\{a, b, c\}$. Therefore, we have $\left(U_{1} \cup\right.$ $\left.U_{2}\right)^{\kappa} \neq U_{1}^{\kappa} \cup U_{2}^{\kappa}$.

(4) Let $U_{1}=\{a, b\}, U_{2}=\{a, c\} \in \mathcal{F}$. Then $\left(U_{1} \cap U_{2}\right)^{\kappa}=\{a\}^{\kappa}=\{a\}$ and

$$
U_{1}^{\kappa} \cap U_{2}^{\kappa}=\{a, b\}^{\kappa} \cap\{a, c\}^{\kappa}=\{a, b, d\} \cap\{a, c, d\}=\{a, d\} .
$$

Therefore, we have $\left(U_{1} \cap U_{2}\right)^{\kappa} \neq U_{1}^{\kappa} \cap U_{2}^{\kappa}$.

\section{3. $(\kappa, \psi)$-continuous and $\left(\mathcal{F}_{\kappa}, \mathcal{G}_{\psi}\right)$-continuous functions}

In this section we consider functions between sets with operations. The sets with operations are denoted by

$$
(X, \mathcal{F}, \kappa),(Y, \mathcal{G}, \psi),(Z, \mathcal{H}, \eta), \ldots \text { and so on. }
$$

Let $(X, \mathcal{F}, \kappa)$ and $(Y, \mathcal{G}, \psi)$ be spaces, that is, $\kappa: \mathcal{F} \rightarrow \mathcal{P}(X)$ and $\psi: \mathcal{G} \rightarrow$ $\mathcal{P}(Y)$ are operations with $\mathcal{F} \subset \mathcal{P}(X)$ and $\mathcal{G} \subset \mathcal{P}(Y)$, respectively.

Definition 3.1. A function $f:(X, \mathcal{F}, \kappa) \rightarrow(Y, \mathcal{G}, \psi)$ is said to be $(\kappa, \psi)$ continuous at $x \in X$ if for each $V \in \mathcal{G}$ containing $f(x)$ there exists $U \in \mathcal{F}$ such that $x \in U$ and $f\left(U^{\kappa}\right) \subset V^{\psi}$. A function $f:(X, \mathcal{F}, \kappa) \rightarrow(Y, \mathcal{G}, \psi)$ is said to be $(\kappa, \psi)$-continuous if it is $(\kappa, \psi)$-continuous at any point $x$ of $X$.

Definition 3.2. A function $f:(X, \mathcal{F}, \kappa) \rightarrow(Y, \mathcal{G}, \psi)$ is said to be $\left(\mathcal{F}_{\kappa}, \mathcal{G}_{\psi}\right)$ continuous at $x \in X$ if for each $\psi$-open set $V$ containing $f(x)$ there exists a $\kappa$-open set $U$ such that $x \in U$ and $f(U) \subset V$. A function $f:(X, \mathcal{F}, \kappa) \rightarrow$ $(Y, \mathcal{G}, \psi)$ is said to be $\left(\mathcal{F}_{\kappa}, \mathcal{G}_{\psi}\right)$-continuous if it is $\left(\mathcal{F}_{\kappa}, \mathcal{G}_{\psi}\right)$-continuous at any point $x$ of $X$.

Remark 3.3. Let $f:(X, \mathcal{F}, \kappa) \rightarrow(Y, \mathcal{G}, \psi)$ be a function and $x \in X$. If there exists no $V \in \mathcal{G}$ containing $f(x)$, then $f$ is $(\kappa, \psi)$-continuous at $x \in X$. If there exists no $V \in \mathcal{G}_{\psi}$ containing $f(x)$, then $f$ is $\left(\mathcal{F}_{\kappa}, \mathcal{G}_{\psi}\right)$-continuous at $x \in X$.

More precisely, we have the following table. 


\begin{tabular}{|c|c|c|}
\hline & condition & at $x$, the function $f$ is \\
\hline (i) & $x \in \cup \mathcal{F}$ and $f(x) \in \cup \mathcal{G}$ & (case by case) \\
\hline (ii) & $x \in \cup \mathcal{F}$ and $f(x) \notin \cup \mathcal{G}$ & $(\kappa, \psi)$-continuous \\
\hline (iii) & $x \notin \cup \mathcal{F}$ and $f(x) \in \cup \mathcal{G}$ & not $(\kappa, \psi)$-continuous \\
\hline (iv) & $x \notin \cup \mathcal{F}$ and $f(x) \notin \cup \mathcal{G}$ & $(\kappa, \psi)$-continuous \\
\hline & condition & at $x$, the function $f$ is \\
\hline (i) & $x \in \cup \mathcal{F}_{\kappa}$ and $f(x) \in \cup \mathcal{G}_{\psi}$ & $($ case by case) \\
\hline (ii) & $x \in \cup \mathcal{F}_{\kappa}$ and $f(x) \notin \cup \mathcal{G}_{\psi}$ & $\left(\mathcal{F}_{\kappa}, \mathcal{G}_{\psi}\right)$-continuous \\
\hline (iii) & $x \notin \cup \mathcal{F}_{\kappa}$ and $f(x) \in \cup \mathcal{G}_{\psi}$ & not $\left(\mathcal{F}_{\kappa}, \mathcal{G}_{\psi}\right)$-continuous \\
\hline (iv) & $x \notin \cup \mathcal{F}_{\kappa}$ and $f(x) \notin \cup \mathcal{G}_{\psi}$ & $\left(\mathcal{F}_{\kappa}, \mathcal{G}_{\psi}\right)$-continuous \\
\hline
\end{tabular}

Example 3.4. The identity function $1_{X}:(X, \mathcal{F}, \kappa) \rightarrow(X, \mathcal{F}, \kappa)$ is both $(\kappa, \kappa)$-continuous and $\left(\mathcal{F}_{\kappa}, \mathcal{F}_{\kappa}\right)$-continuous for any space $(X, \mathcal{F}, \kappa)$.

Proposition 3.5. (1) If a function $f:(X, \mathcal{F}, \kappa) \rightarrow(Y, \mathcal{G}, \psi)$ is $(\kappa, \psi)$ continuous and a function $g:(Y, \mathcal{G}, \psi) \rightarrow(Z, \mathcal{H}, \eta)$ is $(\psi, \eta)$-continuous, then the composite

is $(\kappa, \eta)$-continuous.

$$
g \circ f:(X, \mathcal{F}, \kappa) \rightarrow(Z, \mathcal{H}, \eta)
$$

(2) If a function $f:(X, \mathcal{F}, \kappa) \rightarrow(Y, \mathcal{G}, \psi)$ is $\left(\mathcal{F}_{\kappa}, \mathcal{G}_{\psi}\right)$-continuous and a function $g:(Y, \mathcal{G}, \psi) \rightarrow(Z, \mathcal{H}, \eta)$ is $\left(\mathcal{G}_{\psi}, \mathcal{H}_{\eta}\right)$-continuous, then the composite

$$
g \circ f:(X, \mathcal{F}, \kappa) \rightarrow(Z, \mathcal{H}, \eta)
$$

is $\left(\mathcal{F}_{\kappa}, \mathcal{H}_{\eta}\right)$-continuous.

Proof. (1) Assume that $x \in X$ and there exists $M \in \mathcal{H}$ which contains $g(f(x))$. Then there exists $V \in \mathcal{G}$ which contains $f(x)$ and $g\left(V^{\psi}\right) \subset M^{\eta}$. Also there exists $U \in \mathcal{F}$ containing $x$ such that $f\left(U^{\kappa}\right) \subset V^{\psi}$. It follows that $(g \circ f)\left(U^{\kappa}\right) \subset M^{\eta}$.

(2) Assume that $x \in X$ and there exists $M \in \mathcal{H}_{\eta}$ which contains $g(f(x))$. Then there exists $V \in \mathcal{G}_{\psi}$ which contains $f(x)$ such that $g(V) \subset M$. Also there exists $U \in \mathcal{F}_{\kappa}$ containing $x$ such that $f(U) \subset V$. It follows that $(g \circ f)(U) \subset M$.

The following theorem is a generalization of 1 Theorem (p. 86) of Kelley [5] or 2.6.2 (p. 36) of Brown [2], etc.

Theorem 3.6. The following conditions are equivalent for a function $f:(X, \mathcal{F}, \kappa) \rightarrow(Y, \mathcal{G}, \psi)$.

(1) $f:(X, \mathcal{F}, \kappa) \rightarrow(Y, \mathcal{G}, \psi)$ is $(\kappa, \psi)$-continuous. 
(2) $f\left(\mathrm{Cl}_{\kappa}(A)\right) \subset \mathrm{Cl}_{\psi}(f(A))$ for any subset $A$ of $(X, \mathcal{F}, \kappa)$.

(3) $\mathrm{Cl}_{\kappa}\left(f^{-1}(B)\right) \subset f^{-1}\left(\mathrm{Cl}_{\psi}(B)\right)$ for any subset $B$ of $(Y, \mathcal{G}, \psi)$.

(4) $f^{-1}\left(\operatorname{Int}_{\psi}(D)\right) \subset \operatorname{Int}_{\kappa}\left(f^{-1}(D)\right)$ for any subset $D$ of $(Y, \mathcal{G}, \psi)$.

Proof. (1) $\Longrightarrow(2)$. Assume that $f:(X, \mathcal{F}, \kappa) \rightarrow(Y, \mathcal{G}, \psi)$ is $(\kappa, \psi)$-continuous. Let $y \in f\left(\mathrm{Cl}_{\kappa}(A)\right)$, then $y=f(x)$ for some $x \in \mathrm{Cl}_{\kappa}(A)$. If there exists $V \in \mathcal{G}$ such that $y \in V$, then there exists $U \in \mathcal{F}$ such that $x \in U$ and $f\left(U^{\kappa}\right) \subset V^{\psi}$. Then we have $U^{\kappa} \cap A \neq \emptyset$, and hence $\emptyset \neq f\left(U^{\kappa} \cap A\right) \subset$ $f\left(U^{\kappa}\right) \cap f(A) \subset V^{\psi} \cap f(A)$. This implies $y \in \mathrm{Cl}_{\psi}(f(A))$. If there exists no $V \in \mathcal{G}$ with $y \in V$, then $y \in \mathrm{Cl}_{\psi}(f(A))$ by definition. Therefore we obtain $f\left(\mathrm{Cl}_{\kappa}(A)\right) \subset \mathrm{Cl}_{\psi}(f(A))$.

$(2) \Longrightarrow(3)$. Let $B$ be a subset of $(Y, \mathcal{G}, \psi)$. By $(2)$, we have

$$
f\left(\mathrm{Cl}_{\kappa}\left(f^{-1}(B)\right)\right) \subset \mathrm{Cl}_{\psi}\left(f\left(f^{-1}(B)\right)\right) \subset \mathrm{Cl}_{\psi}(B) .
$$

It follows that $\mathrm{Cl}_{\kappa}\left(f^{-1}(B)\right) \subset f^{-1}\left(\mathrm{Cl}_{\psi}(B)\right)$.

$(3) \Longrightarrow(4)$. Let $D$ be a subset of $(Y, \mathcal{G}, \psi)$. Then by [6, Proposition 3.13] we have

$$
\begin{gathered}
X-\operatorname{Int}_{\kappa}\left(f^{-1}(D)\right)=\mathrm{Cl}_{\kappa}\left(X-f^{-1}(D)\right)=\mathrm{Cl}_{\kappa}\left(f^{-1}(Y-D)\right) \\
\subset f^{-1}\left(\mathrm{Cl}_{\psi}(Y-D)\right)=f^{-1}\left(Y-\operatorname{Int}_{\psi}(D)\right)=X-f^{-1}\left(\operatorname{Int}_{\psi}(D)\right) .
\end{gathered}
$$

It follows that $\operatorname{Int}_{\kappa}\left(f^{-1}(D)\right) \supset f^{-1}\left(\operatorname{Int}_{\psi}(D)\right)$.

$(4) \Longrightarrow(1)$. Let $x \in X$ be any point and $f(x) \in V$ for some $V \in \mathcal{G}$. We see that $V \subset \operatorname{Int}_{\psi}\left(V^{\psi}\right)$ by the definition of $\operatorname{Int}_{\psi}\left(V^{\psi}\right)$ and hence $f(x) \in \operatorname{Int}_{\psi}\left(V^{\psi}\right)$. By (4) we see that

$$
x \in f^{-1}\left(\operatorname{Int}_{\psi}\left(V^{\psi}\right)\right) \subset \operatorname{Int}_{\kappa}\left(f^{-1}\left(V^{\psi}\right)\right) .
$$

Hence there exists $U \in \mathcal{F}$ such that $x \in U \subset U^{\kappa} \subset f^{-1}\left(V^{\psi}\right)$. It follows that $f\left(U^{\kappa}\right) \subset V^{\psi}$. Therefore, $f$ is $(\kappa, \psi)$-continuous at $x \in X$.

Theorem 3.7. Let $f:(X, \mathcal{F}, \kappa) \rightarrow(Y, \mathcal{G}, \psi)$ be a $(\kappa, \psi)$-continuous function. Then the following hold:

(1) $f^{-1}(G)$ is $\kappa$-open in $(X, \mathcal{F}, \kappa)$ for any $\psi$-open set $G$ in $(Y, \mathcal{G}, \psi)$;

(2) $f^{-1}(K)$ is $\kappa$-closed in $(X, \mathcal{F}, \kappa)$ for any $\psi$-closed set $K$ in $(Y, \mathcal{G}, \psi)$.

Proof. (1) Let $G$ be a $\psi$-open set in $Y$. For any $x \in f^{-1}(G)$, we see that $f(x) \in G$, and hence there exists $V \in \mathcal{G}$ such that $f(x) \in V \subset V^{\psi} \subset$ $G$. Since $f$ is $(\kappa, \psi)$-continuous, there exists $U \in \mathcal{F}$ such that $x \in U$ and $f\left(U^{\kappa}\right) \subset V^{\psi} \subset G$. It follows that $x \in U \subset U^{\kappa} \subset f^{-1}(G)$. Hence $f^{-1}(G)$ is $\kappa$-open.

(2) Let $K$ be a $\psi$-closed set in $(Y, \psi)$, namely, $\mathrm{Cl}_{\psi}(K)=K$ by [6, Proposition 3.22]. By Theorem 3.6, the relation

$$
f\left(\mathrm { Cl } _ { \kappa } ( f ^ { - 1 } ( K ) ) \subset \mathrm { Cl } _ { \psi } \left(f\left(f^{-1}(K)\right) \subset \mathrm{Cl}_{\psi}(K)=K\right.\right.
$$


holds. Then we have $\mathrm{Cl}_{\kappa}\left(f^{-1}(K)\right) \subset f^{-1}(K)$ and hence $f^{-1}(K)=$ $\mathrm{Cl}_{\kappa}\left(f^{-1}(K)\right)$, that is, $f^{-1}(K)$ is $\kappa$-closed set in $(X, \kappa)$ by [6, Proposition 3.22].

Remark 3.8. Conditions (1) and (2) in Theorem 3.7 are equivalent by Theorem 3.9.

The following theorem is also a generalization of 1 Theorem (p. 86) of Kelley [5] or 2.6.2 (p. 36) of Brown [2], etc.

Theorem 3.9. The following conditions are equivalent for a function $f:(X, \mathcal{F}, \kappa) \rightarrow(Y, \mathcal{G}, \psi)$.

(1) $f:(X, \mathcal{F}, \kappa) \rightarrow(Y, \mathcal{G}, \psi)$ is $\left(\mathcal{F}_{\kappa}, \mathcal{G}_{\psi}\right)$-continuous.

(2) $f^{-1}(G)$ is $\kappa$-open in $(X, \mathcal{F}, \kappa)$ for any $\psi$-open set $G$ of $(Y, \mathcal{G}, \psi)$.

(3) $f^{-1}(K)$ is $\kappa$-closed in $(X, \mathcal{F}, \kappa)$ for any $\psi$-closed set $K$ of $(Y, \mathcal{G}, \psi)$.

(4) $f\left(\mathcal{F}_{\kappa}-\mathrm{Cl}(A)\right) \subset \mathcal{G}_{\psi}-\mathrm{Cl}(f(A))$ for any subset $A$ of $(X, \mathcal{F}, \kappa)$.

(5) $\mathcal{F}_{\kappa}-\mathrm{Cl}\left(f^{-1}(B)\right) \subset f^{-1}\left(\mathcal{G}_{\psi}-\mathrm{Cl}(B)\right)$ for any subset $B$ of $(Y, \mathcal{G}, \psi)$.

(6) $f^{-1}\left(\mathcal{G}_{\psi}-\operatorname{Int}(D)\right) \subset \mathcal{F}_{\kappa}-\operatorname{Int}\left(f^{-1}(D)\right)$ for any subset $D$ of $(Y, \mathcal{G}, \psi)$.

Proof. (1) $\Longrightarrow(2)$. Assume that $f:(X, \mathcal{F}, \kappa) \rightarrow(Y, \mathcal{G}, \psi)$ is $\left(\mathcal{F}_{\kappa}, \mathcal{G}_{\psi}\right)$ continuous and $G$ is a $\psi$-open set in $(Y, \mathcal{G}, \psi)$. For any $x \in f^{-1}(G)$, we have $f(x) \in G$, and there exists $U \in \mathcal{F}_{\kappa}$ such that $x \in U$ and $f(x) \in f(U) \subset G$ by (1). It follows that $x \in U \subset f^{-1}(G)$ and hence there exists $V \in \mathcal{F}$ such that $x \in V \subset V^{\kappa} \subset U \subset f^{-1}(G)$. Hence $f^{-1}(G)$ is $\kappa$-open in $(X, \mathcal{F}, \kappa)$.

$(2) \Longleftrightarrow(3)$ is obtained by the definitions since $K$ is $\psi$-closed if and only if $X-K$ is $\psi$-open.

$(3) \Longrightarrow(4)$. Let $A$ be a subset of $(X, \mathcal{F}, \kappa)$. Then $\mathcal{G}_{\psi}$ - $\mathrm{Cl}(f(A))$ is $\psi$-closed in $(Y, \mathcal{G}, \psi)$ by Proposition 3.14 of $[6]$. Hence $f^{-1}\left(\mathcal{G}_{\psi^{-}} \mathrm{Cl}(f(A))\right)$ is $\kappa$-closed in $(X, \mathcal{F}, \kappa)$ by $(3)$ and we have

$$
\mathcal{F}_{\kappa^{-}} \mathrm{Cl}\left(f^{-1}\left(\mathcal{G}_{\psi^{-}} \mathrm{Cl}(f(A))\right)\right)=f^{-1}\left(\mathcal{G}_{\psi^{-}} \mathrm{Cl}(f(A))\right) \supset f^{-1}(f(A)) \supset A .
$$

It follows by Proposition 3.16(2) of [6] that

$$
\mathcal{F}_{\kappa^{-}} \mathrm{Cl}(A) \subset \mathcal{F}_{\kappa^{-}} \mathrm{Cl}\left(f^{-1}\left(\mathcal{G}_{\psi^{-}} \mathrm{Cl}(f(A))\right)\right)=f^{-1}\left(\mathcal{G}_{\psi^{-}} \mathrm{Cl}(f(A))\right) .
$$

We conclude that $f\left(\mathcal{F}_{\kappa}-\mathrm{Cl}(A)\right) \subset \mathcal{G}_{\psi^{-}} \mathrm{Cl}(f(A))$.

$(4) \Longrightarrow(5)$. Let $B$ be a subset of $(Y, \mathcal{G}, \psi)$. By (4) we have

$$
f\left(\mathcal{F}_{\kappa^{-}} \mathrm{Cl}\left(f^{-1}(B)\right)\right) \subset \mathcal{G}_{\psi^{-}} \mathrm{Cl}\left(f\left(f^{-1}(B)\right)\right) \subset \mathcal{G}_{\psi^{-}} \mathrm{Cl}(B) .
$$

Therefore we have $\mathcal{F}_{\kappa^{-}} \mathrm{Cl}\left(f^{-1}(B)\right) \subset f^{-1}\left(\mathcal{G}_{\psi^{-}} \mathrm{Cl}(B)\right)$.

$(5) \Longrightarrow(6)$. Let $D$ be a subset of $(Y, \mathcal{G}, \psi)$. Then by [6, Proposition 3.13] and (5) we have

$$
\begin{aligned}
X-\mathcal{F}_{\kappa}-\operatorname{Int}\left(f^{-1}(D)\right) & =\mathcal{F}_{\kappa^{-}} \operatorname{Cl}\left(X-f^{-1}(D)\right) \\
& =\mathcal{F}_{\kappa^{-}} \operatorname{Cl}\left(f^{-1}(Y-D)\right) \subset f^{-1}\left(\mathcal{G}_{\psi^{-}} \mathrm{Cl}(Y-D)\right) \\
& =f^{-1}\left(Y-\mathcal{G}_{\psi^{-}} \operatorname{Int}(D)\right)=X-f^{-1}\left(\mathcal{G}_{\psi^{-}} \operatorname{Int}(D)\right) .
\end{aligned}
$$


It follows that $\mathcal{F}_{\kappa}-\operatorname{Int}\left(f^{-1}(D)\right) \supset f^{-1}\left(\mathcal{G}_{\psi}-\operatorname{Int}(D)\right)$.

(6) $\Longrightarrow(1)$. Let $x \in X$ be any point and $f(x) \in V$ for some $V \in \mathcal{G}_{\psi}$. We see that $\mathcal{G}_{\psi}-\operatorname{Int}(V)=V$ by [6, Proposition 3.22] since $V$ is a $\psi$-open set. Then by (6) we have

$$
f^{-1}(V)=f^{-1}\left(\mathcal{G}_{\psi^{-}} \operatorname{Int}(V)\right) \subset \mathcal{F}_{\kappa^{-}} \operatorname{Int}\left(f^{-1}(V)\right) .
$$

It follows that $f^{-1}(V)=\mathcal{F}_{\kappa}$ - $\operatorname{Int}\left(f^{-1}(V)\right)$, that is, $f^{-1}(V)$ is a $\kappa$-open set by [6, Proposition 3.22]. If we set $U=f^{-1}(V)$, then we have $x \in U=f^{-1}(V)$ and $f(U) \subset V$. Therefore, $f$ is $\left(\mathcal{F}_{\kappa}, \mathcal{G}_{\psi}\right)$-continuous at any point $x \in X$.

Remark 3.10. If $f:(X, \mathcal{F}, \kappa) \rightarrow(Y, \mathcal{G}, \psi)$ is $(\kappa, \psi)$-continuous, then $f$ is $\left(\mathcal{F}_{\kappa}, \mathcal{G}_{\psi}\right)$-continuous by Theorems 3.7 and 3.9. But if $f:(X, \mathcal{F}, \kappa) \rightarrow$ $(Y, \mathcal{G}, \psi)$ is $\left(\mathcal{F}_{\kappa}, \mathcal{G}_{\psi}\right)$-continuous, then $f$ is not always $(\kappa, \psi)$-continuous. Consider the following example. Let $X=\{a, b, c\}$.

(1) Let $\mathcal{F}=\{\{a\},\{b\},\{c\}\}$. We define an operation $\kappa: \mathcal{F} \rightarrow \mathcal{P}(X)$ by $\{a\}^{\kappa}=\{b\}^{\kappa}=\{c\}^{\kappa}=X$. Then $\mathcal{F}_{\kappa}=\{\emptyset, X\}$.

(2) (Example 2.6(1) of [6]) Let $\mathcal{G}=\{\{a\},\{b\},\{a, b\}\}$. We define an operation $\psi: \mathcal{G} \rightarrow \mathcal{P}(X)$ by $\{a\}^{\psi}=\{a, c\},\{b\}^{\psi}=\{b, c\}$ and $\{a, b\}^{\psi}=X$. Then $\mathcal{G}_{\psi}=\{\emptyset\}$.

Then we see $f=1_{X}:(X, \mathcal{F}) \rightarrow(X, \mathcal{G})$ is $\left(\mathcal{F}_{\kappa}, \mathcal{G}_{\psi}\right)$-continuous, but not $(\kappa, \psi)$ continuous (at $a$ and $b$ ).

\subsection{Homeomorphisms between sets with operations.}

Definition 3.11. Let $(X, \mathcal{F}, \kappa)$ and $(Y, \mathcal{G}, \psi)$ be spaces. A $(\kappa, \psi)$-continuous function $f:(X, \mathcal{F}, \kappa) \rightarrow(Y, \mathcal{G}, \psi)$ is called a $(\kappa, \psi)$-homeomorphism if there exists a $(\psi, \kappa)$-continuous function $g:(Y, \mathcal{G}, \psi) \rightarrow(X, \mathcal{F}, \kappa)$ such that $g \circ f=1_{X}$ and $f \circ g=1_{Y}$.

An $\left(\mathcal{F}_{\kappa}, \mathcal{G}_{\psi}\right)$-continuous function $f:(X, \mathcal{F}, \kappa) \rightarrow(Y, \mathcal{G}, \psi)$ is called an $\left(\mathcal{F}_{\kappa}, \mathcal{G}_{\psi}\right)$-homeomorphism if there exists a $\left(\mathcal{G}_{\psi}, \mathcal{F}_{\kappa}\right)$-continuous function $g:(Y, \mathcal{G}, \psi) \rightarrow(X, \mathcal{F}, \kappa)$ such that $g \circ f=1_{X}$ and $f \circ g=1_{Y}$.

By Theorem 3.6 we have the following results. The proof is omitted since they are obtained by standard arguments without difficulty.

Proposition 3.12. The following conditions are equivalent for a bijection $f:(X, \mathcal{F}, \kappa) \rightarrow(Y, \mathcal{G}, \psi)$.

(1) $f:(X, \mathcal{F}, \kappa) \rightarrow(Y, \mathcal{G}, \psi)$ is a $(\kappa, \psi)$-homeomorphism.

(2) $f\left(\mathrm{Cl}_{\kappa}(A)\right)=\mathrm{Cl}_{\psi}(f(A))$ for any subset $A$ of $(X, \mathcal{F}, \kappa)$.

(3) $\mathrm{Cl}_{\kappa}\left(f^{-1}(B)\right)=f^{-1}\left(\mathrm{Cl}_{\psi}(B)\right)$ for any subset $B$ of $(Y, \mathcal{G}, \psi)$.

(4) $f^{-1}\left(\operatorname{Int}_{\psi}(D)\right)=\operatorname{Int}_{\kappa}\left(f^{-1}(D)\right)$ for any subset $D$ of $(Y, \mathcal{G}, \psi)$.

Making use of Theorem 3.9 we have the following results by standard arguments and the proof is omitted. 
Proposition 3.13. The following conditions are equivalent for a bijection $f:(X, \mathcal{F}, \kappa) \rightarrow(Y, \mathcal{G}, \psi)$.

(1) $f:(X, \mathcal{F}, \kappa) \rightarrow(Y, \mathcal{G}, \psi)$ is $\left(\mathcal{F}_{\kappa}, \mathcal{G}_{\psi}\right)$-homeomorphism.

(2) $f^{-1}(G)$ is $\kappa$-open in $(X, \mathcal{F}, \kappa)$ if and only if $G$ is $\psi$-open in $(Y, \mathcal{G}, \psi)$.

(3) $f^{-1}(K)$ is $\kappa$-closed in $(X, \mathcal{F}, \kappa)$ if and only if $K$ is $\psi$-closed in $(Y, \mathcal{G}, \psi)$.

(4) $f\left(\mathcal{F}_{\kappa}-\mathrm{Cl}(A)\right)=\mathcal{G}_{\psi}-\mathrm{Cl}(f(A))$ for any subset $A$ of $(X, \mathcal{F}, \kappa)$.

(5) $\mathcal{F}_{\kappa}-\mathrm{Cl}\left(f^{-1}(B)\right)=f^{-1}\left(\mathcal{G}_{\psi}-\mathrm{Cl}(B)\right)$ for any subset $B$ of $(Y, \mathcal{G}, \psi)$.

(6) $f^{-1}\left(\mathcal{G}_{\psi}-\operatorname{Int}(D)\right)=\mathcal{F}_{\kappa}-\operatorname{Int}\left(f^{-1}(D)\right)$ for any subset $D$ of $(Y, \mathcal{G}, \psi)$.

\section{Subspaces}

Let $(X, \mathcal{F}, \kappa)$ be a space and let $S$ be a subset of $X$. Let

$$
\mathcal{F} \mid S=\{U \cap S \mid U \in \mathcal{F}\} .
$$

Then we try to define

$$
\kappa_{S}: \mathcal{F} \mid S \rightarrow \mathcal{P}(S)
$$

by $\kappa_{S}(U \cap S)=\kappa(U) \cap S$ for any $U \cap S \in \mathcal{F} \mid S$. However, it is not well defined, since $U \cap S=V \cap S$ does not always imply $\kappa(U) \cap S=\kappa(V) \cap S$.

The following is an example which shows that $\kappa_{S}: \mathcal{F} \mid S \rightarrow \mathcal{P}(S)$ is not well defined by $\kappa_{S}(U \cap S)=\kappa(U) \cap S$ for any $U \cap S \in \mathcal{F} \mid S$.

Example 4.1. Consider the example in Remark 2.5.

(1) $S=\{b, c\}:\{b\},\{a, b\} \in \mathcal{F}$ and $\{b\} \cap S=\{b\}=\{a, b\} \cap S$. But

$$
\begin{gathered}
\kappa(\{b\}) \cap S=\{b, c\} \cap S=S \cap S=S \text { and } \\
\kappa(\{a, b\}) \cap S=\{a, b, d\} \cap S=\{b\} .
\end{gathered}
$$

(2) $S=\{a, d\}:\{a\},\{a, b\} \in \mathcal{F}$ and $\{a\} \cap S=\{a\}=\{a, b\} \cap S$. But $\kappa(\{a\}) \cap S=\{a\} \cap S=\{a\}$ and

$$
\kappa(\{a, b\}) \cap S=\{a, b, d\} \cap S=\{a, d\}=S .
$$

Remark 4.2. Let $(X, \tau)$ be a topological space, $S$ a subset of $X$ and $\alpha: \tau \rightarrow$ $\mathcal{P}(X)$ an operation on $\tau$. Let $\tau_{S}$ be the relative topology on $S$. Rosas and Vielma [8] noticed that an operation (called operator in [8]) on a subspace $\alpha_{S}: \tau_{S} \rightarrow \mathcal{P}(S)$ satisfying $\alpha_{S}(G \cap S)=\alpha(G) \cap S$ with $G \in \tau$ is not necessarily well defined in some cases and gave an example just before Theorem 6 of [8]. An operation $\alpha: \tau \rightarrow \mathcal{P}(X)$ is said to be stable with respect to $S$ if $\alpha$ induces an operation $\alpha_{S}: \tau_{S} \rightarrow \mathcal{P}(S)$ satisfying $\alpha_{S}(G \cap S)=\alpha(G) \cap S$ for every $G \in \tau$ (Definition1 of [8]).

Therefore, we adopt the following definition for "subspace".

Definition 4.3. Let $(X, \mathcal{F}, \kappa)$ be a space and $S$ a subset of $X$. Let $\mathcal{F}_{\kappa} \mid S=\left\{U \cap S \mid U \in \mathcal{F}_{\kappa}\right\}$. The triple $\left(S, \mathcal{F}_{\kappa} \mid S\right.$, id $)$ is called a subspace of $(X, \mathcal{F}, \kappa)$, where id: $\mathcal{F}_{\kappa} \mid S \rightarrow \mathcal{P}(S)$ is defined by id $(U)=U$ for any $U \in \mathcal{F}_{\kappa} \mid S$. If $U \in \mathcal{F}_{\kappa}$, then $U \cap S$ is called an $\mathcal{F}_{\kappa} \mid S$-open set of the subspace $\left(S, \mathcal{F}_{\kappa} \mid S\right.$, id) and $(X-U) \cap S$ is called an $\mathcal{F}_{\kappa} \mid S$-closed set of the subspace $\left(S, \mathcal{F}_{\kappa} \mid S\right.$, id). 
Let $S$ and $T$ be subsets of $(X, \mathcal{F}, \kappa)$ and $(Y, \mathcal{G}, \psi)$, respectively. A function $f:\left(S, \mathcal{F}_{\kappa} \mid S\right.$, id $) \rightarrow\left(T, \mathcal{G}_{\psi} \mid T\right.$, id $)$ is said to be $\left(\mathcal{F}_{\kappa}\left|S, \mathcal{G}_{\psi}\right| T\right)$-continuous if $f^{-1}(V) \in \mathcal{F}_{\kappa} \mid S$ for any $V \in \mathcal{G}_{\psi} \mid T$.

Proposition 4.4. Let $(X, \mathcal{F}, \kappa)$ be a space and $S$ a subset of $X$. Let $i^{S}: S \rightarrow X$ be the inclusion function. Then $i^{S}:\left(S, \mathcal{F}_{\kappa} \mid S\right.$, id $) \rightarrow(X, \mathcal{F}, \kappa)$ is $\left(\mathcal{F}_{\kappa} \mid S, \mathcal{F}_{\kappa}\right)$-continuous.

Proof. Since $\left(i^{S}\right)^{-1}(U)=U \cap S \in \mathcal{F}_{\kappa} \mid S$ for any $U \in \mathcal{F}_{\kappa}$, we have the result.

Proposition 4.5. Let $S, T$ and $R$ be subsets of $(X, \mathcal{F}, \kappa),(Y, \mathcal{G}, \psi)$ and $(Z, \mathcal{H}, \eta)$, respectively. Let $f:\left(S, \mathcal{F}_{\kappa} \mid S\right.$, id $) \rightarrow\left(T, \mathcal{G}_{\psi} \mid T\right.$, id $)$ be $\left(\mathcal{F}_{\kappa}\left|S, \mathcal{G}_{\psi}\right| T\right)$ continuous and $g:\left(T, \mathcal{G}_{\psi} \mid T, \mathrm{id}\right) \rightarrow\left(R, \mathcal{H}_{\eta} \mid R, \mathrm{id}\right)$ be $\left(\mathcal{G}_{\psi}\left|T, \mathcal{H}_{\eta}\right| R\right)$-continuous. Then, the composite

$$
g \circ f:\left(S, \mathcal{F}_{\kappa} \mid S, \text { id }\right) \rightarrow\left(T, \mathcal{G}_{\psi} \mid T, \text { id }\right) \rightarrow\left(R, \mathcal{H}_{\eta} \mid R, \text { id }\right)
$$

is $\left(\mathcal{F}_{\kappa}\left|S, \mathcal{H}_{\eta}\right| R\right)$-continuous.

Proof. For any $V \in \mathcal{H}_{\eta} \mid R$, we have $g^{-1}(V) \in \mathcal{G}_{\psi} \mid T$ and therefore $(g \circ$ $f)^{-1}(V)=f^{-1}\left(g^{-1}(V)\right) \in \mathcal{F}_{\kappa} \mid S$. Hence, $g \circ f$ is $\left(\mathcal{F}_{\kappa}\left|S, \mathcal{H}_{\eta}\right| R\right)$-continuous.

We have the following result by the definition of subspace and Propositions 4.4 and 4.5 .

Proposition 4.6. Let $(X, \mathcal{F}, \kappa)$ and $(Y, \mathcal{G}, \psi)$ be spaces. Let $S$ and $T$ be subsets of $X$ and $Y$, respectively. Let $i^{T}: T \rightarrow Y$ be the inclusion function and $f: S \rightarrow Y$ a function with the image $f(S) \subset T$. We write $f_{T}=f: S \rightarrow$ $T$ with the range $T$.

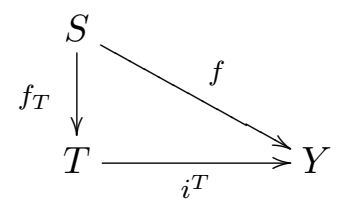

Then the function $f_{T}:\left(S, \mathcal{F}_{\kappa} \mid S\right.$, id $) \rightarrow\left(T, \mathcal{G}_{\psi} \mid T\right.$, id $)$ is $\left(\mathcal{F}_{\kappa}\left|S, \mathcal{G}_{\psi}\right| T\right)$-continuous if and only if the composite $f=i^{T} \circ f_{T}:\left(S, \mathcal{F}_{\kappa} \mid S, \mathrm{id}\right) \rightarrow(Y, \mathcal{G}, \psi)$ is $\left(\mathcal{F}_{\kappa} \mid S, \mathcal{G}_{\psi}\right)$ continuous.

\section{5. $\mathcal{F}_{\kappa}$-connected spaces}

Definition 5.1. Let $(X, \mathcal{F}, \kappa)$ be a space. A subset $S$ of $X$ is said to be $\mathcal{F}_{\kappa}$-connected if $\kappa$-open sets $U$ and $V$ with the following properties do not exist:

$$
U \cap S \neq \emptyset, \quad V \cap S \neq \emptyset, \quad S \subset U \cup V \text { and } U \cap V \cap S=\emptyset .
$$

Remark 5.2. Let $(X, \mathcal{F}, \kappa)$ be a space and $S$ a subset of $X$. Then $S$ is $\mathcal{F}_{\kappa}$-connected if and only if the condition 


$$
U \cap S \neq \emptyset, V \cap S \neq \emptyset \quad \text { and } \quad(U \cap S) \cup(V \cap S)=S
$$

for $\kappa$-open sets $U$ and $V$ implies $(U \cap S) \cap(V \cap S) \neq \emptyset$.

Example 5.3. Let $(X, \mathcal{F}, \kappa)$ be a space and $S$ a subset of $X$. If $S \not \subset \cup \mathcal{F}_{\kappa}$,

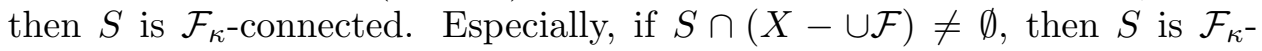
connected.

Remark 5.4. Let $(X, \mathcal{F}, \kappa)$ be a space and $S$ a subset of $X$. If $S$ is not $\mathcal{F}_{\kappa}$-connected, then $S$ is a disjoint union of non-empty $\mathcal{F}_{\kappa} \mid S$-open sets $U \cap S$ and $V \cap S$ : Assume that there exist $\kappa$-open sets $U$ and $V$ which satisfy the following:

$U \cap S \neq \emptyset, V \cap S \neq \emptyset$ and $(U \cap S) \cup(V \cap S)=S$ and $(U \cap S) \cap(V \cap S)=\emptyset$.

Then $X-U$ and $X-V$ are $\kappa$-closed sets and $(X-U) \cap S \subset V \cap S$ and $(X-V) \cap S \subset U \cap S$.

Moreover, we see $S \subset U \cup V$ and $S \subset X-(U \cap V)$. Then we have

$((X-U) \cap S) \cup((X-V) \cap S)=((X-U) \cup(X-V)) \cap S=(X-(U \cap V)) \cap S=S$; $((X-U) \cap S) \cap((X-V) \cap S)=((X-U) \cap(X-V)) \cap S=(X-(U \cup V)) \cap S=\emptyset$.

It follows that $(X-U) \cap S=V \cap S$ and $(X-V) \cap S=U \cap S$. Therefore, $V \cap S$ and $U \cap S$ are $\mathcal{F}_{\kappa} \mid S$-open sets and $\mathcal{F}_{\kappa} \mid S$-closed sets.

Theorem 5.5. Let $f:(X, \mathcal{F}, \kappa) \rightarrow(Y, \mathcal{G}, \psi)$ be $\left(\mathcal{F}_{\kappa}, \mathcal{G}_{\psi}\right)$-continuous and $S \subset X$. If $S$ is $\mathcal{F}_{\kappa}$-connected, then $f(S)$ is $\mathcal{G}_{\psi}$-connected.

Proof. Assume that $f(S)$ is not $\mathcal{G}_{\psi}$-connected. Then there exist $\psi$-open sets $U$ and $V$ of $(Y, \mathcal{G}, \psi)$ which satisfy the following properties:

$$
U \cap f(S) \neq \emptyset, \quad V \cap f(S) \neq \emptyset, \quad U \cap V \cap f(S)=\emptyset \text { and } f(S) \subset U \cup V .
$$

The inverse images $f^{-1}(U)$ and $f^{-1}(V)$ are $\kappa$-open in $(X, \mathcal{F}, \kappa)$ by Theorem 3.9 and satisfy the following properties:

$$
\begin{gathered}
f^{-1}(U) \cap S \neq \emptyset, \quad f^{-1}(V) \cap S \neq \emptyset, \quad f^{-1}(U) \cap f^{-1}(V) \cap S=\emptyset \text { and } \\
S \subset f^{-1}(U) \cup f^{-1}(V) .
\end{gathered}
$$

This contradicts the assumption that $S$ is $\mathcal{F}_{\kappa}$-connected, and hence $f(S)$ must be $\mathcal{G}_{\psi}$-connected.

Theorem 5.6. Let $(X, \mathcal{F}, \kappa)$ be a space. Let $S$ and $T$ be subsets of $X$. If $S$ is $\mathcal{F}_{\kappa}$-connected and $S \subset T \subset \mathcal{F}_{\kappa}-\mathrm{Cl}(S)$, then $T$ is $\mathcal{F}_{\kappa}$-connected.

Proof. The proof is done by a standard argument as follows. Assume that $T$ is not $\mathcal{F}_{\kappa}$-connected. Then there exist $\kappa$-open sets $U$ and $V$ which satisfy the following:

$U \cap T \neq \emptyset, V \cap T \neq \emptyset$ and $(U \cap T) \cup(V \cap T)=T$ and $(U \cap T) \cap(V \cap T)=\emptyset$. 
If $U \cap S=\emptyset$, then $S \subset X-U$ and hence

$$
T \subset \mathcal{F}_{\kappa^{-}} \mathrm{Cl}(S) \subset \mathcal{F}_{\kappa^{-}} \mathrm{Cl}(X-U)=X-U
$$

by Proposition 3.22 of [6], which implies $U \cap T=\emptyset$. Therefore, $U \cap S \neq \emptyset$. Similarly, we have $V \cap S \neq \emptyset$. Then $S$ is not $\mathcal{F}_{\kappa}$-connected, which contradicts the assumption. Hence $T$ must be $\mathcal{F}_{\kappa}$-connected.

Theorem 5.7. Let $(X, \mathcal{F}, \kappa)$ be a space and $S_{\lambda}$ a subset of $X$ for each $\lambda \in \Lambda$. If $S_{\lambda}$ is $\mathcal{F}_{\kappa}$-connected for each $\lambda \in \Lambda$ and $\bigcap_{\lambda \in \Lambda} S_{\lambda} \neq \emptyset$, then $\bigcup_{\lambda \in \Lambda} S_{\lambda}$ is $\mathcal{F}_{\kappa}$-connected.

Proof. Let us write $S=\bigcup_{\lambda \in \Lambda} S_{\lambda}$. Assume that $S$ is not $\mathcal{F}_{\kappa}$-connected. Then there exist $\kappa$-open sets $U$ and $V$ which satisfy the following:

$U \cap S \neq \emptyset, V \cap S \neq \emptyset$ and $(U \cap S) \cup(V \cap S)=S$ and $(U \cap S) \cap(V \cap S)=\emptyset$.

There exists an element $x \in \bigcap_{\lambda \in \Lambda} S_{\lambda} \subset S$. One of $U \cap S$ and $V \cap S$, say $U \cap S$, contains $x$. Then $x \notin V \cap S$ and the relation $V \cap S \neq \emptyset$ implies that there exists $\lambda \in \Lambda$ such that $V \cap S_{\lambda} \neq \emptyset$. Moreover, $x \in U \cap S_{\lambda} \neq \emptyset$, $\left(U \cap S_{\lambda}\right) \cup\left(V \cap S_{\lambda}\right)=S_{\lambda}$ and $\left(U \cap S_{\lambda}\right) \cap\left(V \cap S_{\lambda}\right) \subset(U \cap S) \cap(V \cap S)=\emptyset$. Therefore, $S_{\lambda}$ is not $\mathcal{F}_{\kappa}$-connected, which is a contradiction.

Theorem 5.8. Let $(X, \mathcal{F}, \kappa)$ be a space and $S$ a subset of $X$ such that $S \subset \cup \mathcal{F}_{\kappa}$. Let $(\{1,2\}, \delta)$ be a topological space of two points $\{1,2\}$ with the discrete topology $\delta$, which is regarded as a space $(\{1,2\}, \delta$,id) as in Example 2.2. Then $S$ is $\mathcal{F}_{\kappa}$-connected if and only if any $\left(\mathcal{F}_{\kappa} \mid S, \delta\right)$-continuous function $f:\left(S, \mathcal{F}_{\kappa} \mid S, \mathrm{id}\right) \rightarrow(\{1,2\}, \delta, \mathrm{id})$ is a constant function.

Proof. We note that any constant function $f:\left(S, \mathcal{F}_{\kappa} \mid S\right.$, id $) \rightarrow(\{1,2\}, \delta$, id $)$ is $\left(\mathcal{F}_{\kappa} \mid S, \delta\right)$-continuous by Theorem 3.9, since $S$ is an $\mathcal{F}_{\kappa} \mid S$-open set by the assumption $S \subset \cup \mathcal{F}_{\kappa}$ (see Proposition 2.8 of [6]).

Assume that $S$ is $\mathcal{F}_{\kappa}$-connected and a $\left(\mathcal{F}_{\kappa} \mid S, \delta\right)$-continuous function $f:\left(S, \mathcal{F}_{\kappa} \mid S\right.$, id $) \rightarrow(\{1,2\}, \delta$, id $)$ is surjective. Let us notice that the domain of $f$ is the set $S$, so $f^{-1}(\{1\}) \cap S=f^{-1}(\{1\})$. We know that $f^{-1}(\{1\})$ is $\mathcal{F}_{\kappa} \mid S$-open by the continuity of $f$, so there exists $U \in \mathcal{F}_{\kappa}$ such that $f^{-1}(\{1\})=U \cap S$. We can do the same with $f^{-1}(\{2\})$. Therefore, $f^{-1}(\{1\})$ and $f^{-1}(\{2\})$ are disjoint $\mathcal{F}_{\kappa} \mid S$-open sets of $S$, that is, $S$ is not $\mathcal{F}_{\kappa}$-connected. Therefore, $f:\left(S, \mathcal{F}_{\kappa} \mid S\right.$, id $) \rightarrow(\{1,2\}, \delta$, id $)$ must be a constant map.

Assume that $S$ is not $\mathcal{F}_{\kappa}$-connected. Then there exist $\kappa$-open sets $U$ and $V$ which satisfy the following (see Remark 5.2):

$U \cap S \neq \emptyset, V \cap S \neq \emptyset$ and $(U \cap S) \cup(V \cap S)=S$ and $(U \cap S) \cap(V \cap S)=\emptyset$.

We define a function $f:\left(S, \mathcal{F}_{\kappa} \mid S\right.$, id $) \rightarrow(\{1,2\}, \delta$, id $)$ by $f(U \cap S)=1$ and $f(V \cap S)=2$. Then $f$ is a surjective $\left(\mathcal{F}_{\kappa} \mid S, \delta\right)$-continuous function. 


\section{6. $\mathcal{F}_{\kappa}$-compact spaces}

Definition 6.1. Let $(X, \mathcal{F}, \kappa)$ be a space. A subset $S$ of $X$ is said to be $\mathcal{F}_{\kappa}$-compact if every $\kappa$-open cover of $S$ has a finite subcover, that is, if $S \subset \bigcup_{\lambda \in \Lambda} U_{\lambda}$ and $U_{\lambda} \in \mathcal{F}_{\kappa}$ for any $\lambda \in \Lambda$ then there exist a finite number of $\lambda_{1}, \lambda_{2}, \ldots, \lambda_{n} \in \Lambda$ such that $S \subset U_{\lambda_{1}} \cup U_{\lambda_{2}} \cup \cdots \cup U_{\lambda_{n}}$.

Remark 6.2. Let $(X, \mathcal{F}, \kappa)$ be a space and $S$ a subset of $X$. If there is no $\kappa$-open cover of $S$, then $S$ is $\mathcal{F}_{\kappa}$-compact. Therefore, if $S \not \subset \cup_{U \in \mathcal{F}_{\kappa}} U$, then $S$ is $\mathcal{F}_{\kappa}$-compact. Especially, if $S \not \subset \cup_{U \in \mathcal{F}} U$, then $S$ is $\mathcal{F}_{\kappa}$-compact.

Let $(X, \mathcal{F}, \kappa)$ be a space. We define:

$$
\kappa(\mathcal{F})=\left\{U^{\kappa} \mid U \in \mathcal{F}\right\} .
$$

Definition 6.3. Let $(X, \mathcal{F}, \kappa)$ be a space. A subset $S$ of $X$ is said to be $\kappa(\mathcal{F})$-compact if every $\kappa(\mathcal{F})$-cover of $S$ has a finite subcover, that is, if $S \subset \bigcup_{\lambda \in \Lambda} U_{\lambda}$ and $U_{\lambda} \in \kappa(\mathcal{F})$ for any $\lambda \in \Lambda$ then there exist a finite number of $\lambda_{1}, \lambda_{2}, \ldots, \lambda_{n} \in \Lambda$ such that $S \subset U_{\lambda_{1}} \cup U_{\lambda_{2}} \cup \cdots \cup U_{\lambda_{n}}$.

Proposition 6.4. Let $(X, \mathcal{F}, \kappa)$ be a space and $S$ a subset of $X$. If $S$ is $\kappa(\mathcal{F})$-compact, then $S$ is $\mathcal{F}_{\kappa}$-compact.

Proof. Every $\kappa$-open set $A$ is written as $A=\cup_{\lambda \in \Lambda} U_{\lambda}=\cup_{\lambda \in \Lambda} U_{\lambda}^{\kappa}$ where $U_{\lambda} \in \mathcal{F}$ for each $\lambda \in \Lambda$ by [6, Proposition 2.4], that is, any $\kappa$-open set is a union of some sets in $\kappa(\mathcal{F})$. Hence every $\kappa$-open cover of $S$ is a $\kappa(\mathcal{F})$-cover of $S$, and hence we have the result by standard arguments.

Theorem 6.5. Let $(X, \mathcal{F}, \kappa)$ be a space and $S$ a subset of $X$. If $S$ is $\mathcal{F}_{\kappa}$-compact and $K$ is a $\mathcal{F}_{\kappa} \mid S$-closed subset of $S$, then $K$ is $\mathcal{F}_{\kappa}$-compact.

Proof. Since $K$ is a $\mathcal{F}_{\kappa} \mid S$-closed subset of $S$, there exist $U \in \mathcal{F}_{\kappa}$ such that $K=S \cap(X-U)$. Then we have $S-K=U \cap S$. Assume that $K \subset \bigcup_{\lambda \in \Lambda} U_{\lambda}$ and $U_{\lambda} \in \mathcal{F}_{\kappa}$ for any $\lambda \in \Lambda$. Then

$$
S=(S-K) \cup K \subset U \cup\left(\bigcup_{\lambda \in \Lambda} U_{\lambda}\right) .
$$

Since $S$ is $\mathcal{F}_{\kappa}$-compact, there exist numbers $\lambda_{1}, \lambda_{2}, \ldots, \lambda_{n} \in \Lambda$ such that

$$
S=(S-K) \cup K \subset U \cup U_{\lambda_{1}} \cup U_{\lambda_{2}} \cup \cdots \cup U_{\lambda_{n}} .
$$

If $x \in K$, then $x \notin U$ and hence $x \in U_{\lambda_{1}} \cup U_{\lambda_{2}} \cup \cdots \cup U_{\lambda_{n}}$. It follows that $K \subset U_{\lambda_{1}} \cup U_{\lambda_{2}} \cup \cdots \cup U_{\lambda_{n}}$. Hence $K$ is $\mathcal{F}_{\kappa}$-compact.

Theorem 6.6. Let $f:(X, \mathcal{F}, \kappa) \rightarrow(Y, \mathcal{G}, \psi)$ be $\left(\mathcal{F}_{\kappa}, \mathcal{G}_{\psi}\right)$-continuous and $S \subset X$. If $S$ is $\mathcal{F}_{\kappa}$-compact, then $f(S)$ is $\mathcal{G}_{\psi}$-compact.

Proof. Assume that $f(S) \subset \bigcup_{\lambda \in \Lambda} U_{\lambda}$ and $U_{\lambda} \in \mathcal{G}_{\psi}$ for any $\lambda \in \Lambda$. Then we have $S \subset \bigcup_{\lambda \in \Lambda} f^{-1}\left(U_{\lambda}\right)$. Since $S$ is $\mathcal{F}_{\kappa}$-compact and $f^{-1}\left(U_{\lambda}\right)$ is $\kappa$-open for each $\lambda \in \Lambda$ by Theorem 3.9, there exist a finite number of $\lambda_{1}, \lambda_{2}, \ldots, \lambda_{n} \in \Lambda$ such that $S \subset f^{-1}\left(U_{\lambda_{1}}\right) \cup f^{-1}\left(U_{\lambda_{2}}\right) \cup \cdots \cup f^{-1}\left(U_{\lambda_{n}}\right)$. It follows that $f(S) \subset$ $U_{\lambda_{1}} \cup U_{\lambda_{2}} \cup \cdots \cup U_{\lambda_{n}}$. 


\section{Acknowledgements}

The second author was supported by JSPS KAKENHI Grant Number JP15K04884.

The authors would like to thank the referee for many useful comments to improve the paper, especially about the definition of subspace and the presentation of the paper.

\section{References}

[1] C. K. Basu, B. M. Uzzal Afsan, and M. K. Ghosh, A Class of functions and separation axioms with respect to an operation, Hacettepe J. Math. Stat. 38 (2009), 103-118.

[2] R. Brown, Topology: A Geometric Account of General Topology, Homotopy Types and the Fundamental Groupoid, Ellis Horwood, Chichester, 1988.

[3] D. S. Janković, On functions with $\alpha$-closed graphs, Glas. Mat. Ser. III 18(38) (1983), 141-148.

[4] S. Kasahara, Operation-compact spaces, Math. Japon. 24 (1979), 97-105.

[5] J. L. Kelley, General Topology, D. Van Nostrand Company, Inc., Toronto-New YorkLondon, 1955.

[6] F. Nakaoka and N. Oda, Interiors and closures in a set with an operation, Commun. Korean Math. Soc. 29 (2014), 555-568.

[7] H. Ogata, Operations on topological spaces and associated topology, Math. Japon. 36 (1991), 175-184.

[8] E. Rosas and J. Vielma, Operator-compact and operator-connected spaces, Scientiae Math. 1 (1998), 203-208.

Department of Applied Mathematics, Fukuoka University, Fukuoka 8140180, JAPAN

E-mail address: fumie@fukuoka-u.ac.jp

E-mail address: odanobu@fukuoka-u.ac.jp 